\title{
ON AUTOMORPHIC-INVERSE PROPERTIES IN LOOPS
}

R. ARTZY

Introduction. In a loop $(G, \cdot)$ we define $J$ as the mapping that takes every element into its right inverse, i.e., $x \cdot x J=1$, for all $x$ in $G$. It is well known [3] that in I.P. loops $J$ is an anti-automorphism. In crossed-inverse loops (in short C.I. loops), which are defined as satisfying either of the equivalent identities $x y \cdot x J=y$ or $x(y \cdot x J)=y$, for all $x$ and $y$ in $G, J$ is known [1] to be an automorphism. On the other hand, easily constructed counter-examples show that a loop in which $J$ is an anti-automorphism is not necessarily I.P., and that a loop in which $J$ is an automorphism is not necessarily C.I. Furthermore a loop which is I.P. everywhere, i.e. all of whose isotopes are I.P., is known [4] to be Moufang. A loop which is C.I. everywhere is an abelian group; this can be easily checked by computation, but it is also obvious, due to the fact that the C.I. property can be represented in a 3 -web by the validity of the Thomsen figure (cf. [7]) in a special position while the general Thomsen figure corresponds to both associativity and commutativity.

It is the purpose of this paper to show that an I.P. loop in which $J$ is an anti-automorphism everywhere is Moufang, and that a C.I. loop in which $J$ is an automorphism everywhere is an abelian group. Thus we show that the weaker "automorphic-inverse properties" $(x y) J=y J \cdot x J$ and $(x y) J=x J \cdot y J$ in I.P. loops and C.I. loops, respectively, are sufficient to replace the full I.P. and C.I. properties of the isotopes.

Theorem 1. An I.P. loop, in all of whose isotopes $J$ is an antiautomorphism, is Moufang.

Proof. We consider an isotope with $x g * y=x y$. Its unit is $g$. Let $x J^{*}$ be the right inverse of $x$ in the isotope. Using the inverse property, which implies $J=J^{-1}$, we have then

$$
x g^{-1} \cdot x J^{*}=g, \text { and } J^{*}=J L(g) R(g) .
$$

The theorem assumes $(x * y) J^{*}=y J^{*} * x J^{*}$, that is $g\left(x g^{-1} \cdot y\right)^{-1} \cdot g$ $=\left[\left(g y^{-1} \cdot g\right) g^{-1}\right]\left(g x^{-1} \cdot g\right)$. With $g x^{-1}=a, y^{-1}=b$ we get $(g \cdot b a) g=g b \cdot a g$, for all $a, b, g$ in the loop. Thus the loop is Moufang.

Presented to the Society, August 29, 1958; received by the editors July 6, 1958 and, in revised form, November 24, 1958. 
Theorem 2. A C.I. loop, $G$, in all of whose isotopes $J$ is an automorphism, is an abelian group.

Proof. The proof will be performed in 5 stages, (i) to (v).

(i) The inverses in $G$ are unique.

Let the isotope be defined by $x g * f y=x y$. Then we have, using the C.I. property,

$$
\left(g J^{-1} \cdot x\right)\left(x J^{*} \cdot f J\right)=f g, \quad \text { and } \quad J^{*}=J L(g) L(f g) L(f) .
$$

The identity $(x * y) J^{*}=x J^{*} * y J^{*}$ becomes

(1) $f\left[f g \cdot\left(g\left((g \cdot x J)\left(y J \cdot f J^{2}\right)\right)\right)\right]=\left[g J^{-1} \cdot(f(f g \cdot(g \cdot x J)))\right][f g \cdot(g \cdot y J)]$.

Putting $x=g^{2} g$ and $y=f=1$, yields $1=\left(g J^{-1} \cdot g J\right) g^{2}$, and finally $g J^{-1}=g J$. Thus inverses are unique in $G$.

(ii) The squares form a normal subloop and lie in the centre.

If we substitute in (1) $g x^{-1}=a, y^{-1} f=b$ we get

$$
f[f g \cdot(g \cdot a b)]=\left[g^{-1}(f(f g \cdot a))\right]\left[f g \cdot\left(g \cdot f^{-1} b\right)\right] .
$$

This is an autotopism (cf. [4])

$$
\left(L(f g) L(f) L\left(g^{-1}\right), L\left(f^{-1}\right) L(g) L(f g), L(g) L(f g) L(f)\right) .
$$

With $f=1$ this becomes

$$
\left(L(g) L\left(g^{-1}\right), L(g) L(g), L(g) L(g)\right) .
$$

According to [2] every autotopism $(U, V, W)$ in a C.I. loop implies another autotopism $(L(1 U), L(1 V), L(1 W))$. Thus (3) implies $\left(I, L\left(g^{2}\right), L\left(g^{2}\right)\right)$ as a new autotopism, that is,

$$
a \cdot g^{2} b=g^{2} \cdot a b .
$$

With $b=1$ this yields $a g^{2}=g^{2} a$, hence the squares of the elements commute with all elements of $G$. Equation (4) becomes $a \cdot b g^{2}=a b \cdot g^{2}$. Thus all the squares belong to the right nucleus, but, as the author has proved [2], all the elements of the right nucleus are centre elements, and therefore all squares of loop elements lie in the centre. Moreover the squares form a normal subloop:

$$
x^{2} y^{2}=x^{2} y^{2}\left(x y \cdot x^{-1} y^{-1}\right)=x y \cdot\left(x^{2} x^{-1} \cdot y^{2} y^{-1}\right)=x y \cdot x y=(x y)^{2},
$$

and $\left(x^{2}\right) J=(x J)^{2}$.

(iii) $G$ is commutative.

According to [2], the elements $1 U$ and $1 \mathrm{~V}$, for all autotopisms ( $U, V, W$ ) of $G$, form a Moufang subloop, and the cubes of the elements of this subloop lie in the centre of $G$. Since also the squares lie in the centre, $1 \mathrm{U}$ and $1 \mathrm{~V}$ themselves lie in the centre. In particular 
take as $(U, V, W)$ the autotopism (2), then $1 U=g^{-1}(f \cdot f g)$ and $1 V$ $=f g \cdot g f^{-1}$ lie in the centre of $G$. Therefore we have

$$
\begin{gathered}
g^{-1}\left(g^{-1} f \cdot 1 V\right)=\left(g^{-1} \cdot g^{-1} f\right) \cdot 1 V, \\
f=g^{-1}\left(g^{-1} f \cdot\left(f g \cdot g f^{-1}\right)\right)=\left(g^{-1} \cdot g^{-1} f\right)\left(f g \cdot g f^{-1}\right), \\
f\left(g \cdot g f^{-1}\right)=f g \cdot g f^{-1}
\end{gathered}
$$

and

$$
\begin{gathered}
1 V \cdot f^{-1} g^{-1}=f^{-1} g^{-1} \cdot 1 V, \\
g f^{-1}=\left(f g \cdot g f^{-1}\right) \cdot f^{-1} g^{-1}=f^{-1} g^{-1} \cdot\left(f g \cdot g f^{-1}\right), \\
g f^{-1} \cdot f g=f g \cdot g f^{-1} .
\end{gathered}
$$

We interchange $f$ and $g$ in (5) and get

$$
g\left(f \cdot f g^{-1}\right)=g f \cdot f g^{-1}=\left(g f^{-1} \cdot f g\right) f^{2} g^{-2} .
$$

In view of (6) this becomes

$$
g^{-1}(f \cdot f g)=\left(f g \cdot g f^{-1}\right) f^{2} g^{-2}, \text { or } 1 U=1 V \cdot f^{2} g^{-2} .
$$

Now obviously $1 \mathrm{~W}=1 \mathrm{U} \cdot 1 \mathrm{~V}$, hence $1 \mathrm{~W}=1 \mathrm{~V} \cdot f^{2} g^{-2} \cdot 1 \mathrm{~V}$, that is, $f(f g \cdot g)=f^{2} g^{-2}\left(f g \cdot g f^{-1}\right)^{2}$, and using the multiplication rule for squares, $f(f g \cdot g)=f^{2} g^{-2} f^{2} g^{2} g^{2} f^{-2}=f^{2} g^{2}$, and $f g=f^{2} g^{2} \cdot g^{-1} f^{-1}=g f$. Thus the commutativity of $G$ is established.

The commutativity and the C.I. property imply the inverse property. Moreover the alternative property holds:

$$
a \cdot a b=a^{2}\left(a \cdot a^{-1} b\right)=a^{2} b .
$$

(iv) $G$ is an $A$-loop.

Using the associativity of multiplication by squares, we may write the autotopism (2)

$$
(U, V, W)=\left(L(f g)^{-1} L(f) L(g), L(f) L(g) L(f g)^{-1}, L(g) L(f g)^{-1} L(f)\right) .
$$

Now $1 U=1 V=1$, and that implies $U=V=W$. Hence $V$ $=L(f) L(g) L(f g)^{-1}$ is an automorphism. We shall prove now that every inner mapping of $G$ is the product of inner mappings of the form $L(f) L(g) L(f g)^{-1}$. Owing to the commutativity of $G$ and the property $L^{-1}(x)=L\left(x^{-1}\right)$ of I.P. loops, every inner mapping $S$ of $G$ has the form $S=\prod_{k=1}^{n} L\left(a_{k}\right)$, with $1 S=1$. We can write

$$
\begin{aligned}
S=\left[L\left(a_{1}\right) L\left(a_{2}\right) L\left(a_{1} a_{2}\right)^{-1}\right] & {\left[L\left(a_{1} a_{2}\right) L\left(a_{3}\right) L\left(a_{1} a_{2} \cdot a_{3}\right)^{-1}\right] } \\
\cdots & {\left[L\left(1 \prod_{k=1}^{n-1} L\left(a_{k}\right)\right) L\left(a_{n}\right) L\left(1 \prod_{k=1}^{n} L\left(a_{k}\right)\right)^{-1}\right] }
\end{aligned}
$$

because the last term of each bracket and the first term of the next 
bracket are of the type $L(x) L\left(x^{-1}\right)$ and cancel out; furthermore the last term of the last bracket is

$$
L\left(1 \prod_{k=1}^{n} L\left(a_{k}\right)\right)^{-1}=L(1 S)^{-1}=L\left(1^{-1}\right)=I .
$$

Each of the brackets contains a product of the form

$$
V=L(f) L(g) L(f g)^{-1}
$$

which is an automorphism. Hence every inner mapping $S$ is a product of automorphisms, and therefore an automorphism. A loop in which every inner mapping is an automorphism is usually called an $A$-loop.

(v) $G$ is abelian.

$G$ is a commutative I.P. loop. $A$-loops with the inverse property are diassociative [5], and if they are also commutative they are Moufang [6]. Thus we have the identity $(x y \cdot x) z=x(y \cdot x z)$, and therefore $x^{2} y \cdot z=x(y \cdot x z), y z=x^{-1}(y \cdot x z)$, and $y z \cdot x=y \cdot z x$. Hence $G$ is associative and therefore abelian.

Remark. In recent work (to appear in Pacific J. Math.) J. M. Osborn has been dealing with a generalization of both C.I. and I.P. loops: "weak-inverse loops" in which $x y \cdot z=1$ if, and only if, $x \cdot y z=1$. In weak-inverse loops $J^{2}$ is an automorphism, but this property is not sufficient for defining weak-inverse loops. Osborn has investigated loops which are weak-inverse everywhere, and these loops have interesting properties. In connection with the present paper it might be of interest whether a weak-inverse loop all of whose isotopes have $J^{2}$ as an automorphism (and are not necessarily weak-inverse) has already the same properties, such that again an "automorphic-inverse property" would suffice to replace the full loop identity in the isotopes.

\section{REFERENCES}

1. R. Artzy, On loops with a special property, Proc. Amer. Math. Soc. vol. 6 (1955) pp. 448-453.

2. - Crossed-inverse and related loops, Trans. Amer. Math. Soc. vol. 91 (1959) pp. 480-492.

3. R. H. Bruck, Some results in the theory of quasigroups, Trans. Amer. Math. Soc. vol. 55 (1944) pp. 19-52.

4. - Contributions to the theory of loops, Trans. Amer. Math. Soc. vol. 60 (1946) pp. 245-354.

5. R. H. Bruck and L. J. Paige, Loops whose inner mappings are automorphisms, Ann. of Math. vol. 63 (1956) pp. 308-323.

6. J. M. Osborn, $A$ theorem on A-loops, Proc. Amer. Math. Soc. vol. 9 (1958) pp. 347-349.

7. G. Pickert, Projektive Ebenen, Berlin-Göttingen-Heidelberg, Springer, 1955.

ISRAEL INSTITUTE OF TECHNOLOGY AND

UNIVERSITY OF WISCONSIN 